Artículo científico

Volumen 32(1):120-136. Enero-abril, 2021

e-ISSN 2215-3608, doi:10.15517/am.v32i1.39284

https://www.revistas.ucr.ac.cr/index.php/agromeso

\title{
Pérdida de suelo en el sistema ancestral wachado en el trópico alto de Nariño, Colombia ${ }^{1}$
}

\section{Soil loss in the wachado old system in the upper tropics of Nariño, Colombia}

\author{
Belisario Volverás-Mambuscay², José Domingo Merchancano-Rosero², Juan Fernando López-Rendón², \\ José Manuel Campo-Quesada²
}

1 Recepción: 18 de noviembre, 2019. Aceptación: 11 de junio, 2020. El trabajo formó parte del proyecto "Ajuste de prácticas culturales para manejo adecuado del sistema de siembra en guachado en la zona alto andina de Nariño", ejecutado por la Corporación Colombiana de investigación Agropecuaria (AGROSAVIA), financiado por el Ministerio de Agricultura y Desarrollo Rural de Colombia.

${ }^{2}$ Corporación Colombiana de investigación Agropecuaria (AGROSAVIA), Centro de Investigación Obonuco, Pasto, Colombia. bvolveras@ agrosavia.co (autor para la correspondencia, https://orcid.org/0000-0002-6571-670X), jdmerchancano@agrosavia.co (https://orcid.org/00000001-5798-9663), jflopezr@agrosavia.co (https://orcid.org/0000-0002-7035-1880), jcampoq@agrosavia.co (https://orcid.org/0000-00019927-6629).

\section{Resumen}

Introducción. El sistema ancestral wachado (surco o camellón), usado en laderas de Nariño para la producción de papa y leche, no utiliza maquinaria pesada y genera menor impacto sobre la productividad del suelo. Objetivo. Evaluar el ajuste de la inclinación del surcado para papa y su efecto sobre la pérdida de suelo en la zona alto andina de Nariño. Materiales y métodos. El trabajo se desarrolló en las localidades de Pasto, Potosí y Pupiales, entre mayo y octubre del 2014. Se utilizó un diseño de bloques completos al azar con tres repeticiones y cuatro tratamientos (wachado a $90^{\circ}, 70^{\circ}, 60^{\circ}$ y labranza comercial). En la base de cada unidad experimental se construyeron canaletas para recoger el suelo desplazado. Se evaluó la pérdida del suelo y rendimiento de tubérculos. Resultados. Se observó efecto significativo de la inclinación de los surcos sobre la pérdida de suelo; la mayor pérdida total de suelo se obtuvo en surcos en sentido de la pendiente $\left(90^{\circ}\right)$, con promedio de $69,29 \mathrm{t} \mathrm{ha}^{-1}$ para labranza comercial y $63,51 \mathrm{t}$ ha ${ }^{-1}$ para wachado; la menor pérdida fue $45,76 \mathrm{t} \mathrm{ha}^{-1}$ en wachado a $60^{\circ}$. De la pérdida total de suelo, la mayor proporción se presentó en los primeros dos meses del cultivo que incluyó siembra y aporques; en estas labores se perdió el $90 \%$, $60 \%$ y $67 \%$ en las localidades de Pasto, Potosí y Pupiales, respectivamente. No se observó efecto de los tratamientos de inclinación del surcado sobre el rendimiento de papa, que alcanzó un promedio de 44,94 t ha ${ }^{-1}$. Conclusión. La reducción de la inclinación de los surcos disminuyó la pérdida de suelo durante el ciclo de cultivo sin afectar el rendimiento y se sugiere validarla a nivel comercial, ya que podría contribuir a la disminución de la degradación del suelo en las laderas andinas de Nariño.

Palabras claves: erosión, laderas, papa, labranza, sostenibilidad.

\section{Abstract}

Introduction. The ancestral wachado (furrow or ridge) system, used on the slopes of Nariño for the production of potatoes and milk, does not use heavy machinery and has less impact on soil productivity. Objective. To evaluate 
the furrow inclination adjustment of the furrow for potatoes and its effect on soil loss in the high Andean zone of Nariño. Materials and methods. The work was carried out in the towns of Pasto, Potosí, and Pupiales between May and October 2014. A complete randomized block design with three repetitions and four treatments (wachado at $90^{\circ}$, $70^{\circ}, 60^{\circ}$, and commercial tillage) was used. Gutters were built at the base of each experimental unit to collect the displaced soil. Soil loss and tuber yield were evaluated. Results. A significant effect of furrow inclination on soil loss was observed; the highest total loss of soil was obtained in furrows in the direction of the slope $\left(90^{\circ}\right)$ with an average of $69.29 \mathrm{t} \mathrm{ha}^{-1}$ for commercial tillage and $63.51 \mathrm{t} \mathrm{ha}^{-1}$ for wachado; the lowest loss was $45.76 \mathrm{t} \mathrm{ha}^{-1}$ in wachado at $60^{\circ}$. Of the total loss of soil, the highest proportion occurred in the first two months of cultivation, which included sowing and hilling; $90 \%, 60 \%$, and $67 \%$ were lost in these tasks for Pasto, Potosí, and Pupiales, respectively. No furrow inclination effect was observed on potato yield, which reached an average of $44.94 \mathrm{t} \mathrm{ha}^{-1}$. Conclusion. The furrows inclination reduction diminished the soil loss during the cultivation cycle without affecting the yield and it is suggested to validate it at a commercial level since it could contribute to the reduction of soil degradation in the Andean slopes of Nariño.

Keywords: erosion, slopes, potato, tillage, sustainability.

\section{Introducción}

Al igual que en muchas regiones del mundo, el cultivo de papa (Solanum tuberosum) es la actividad agrícola más sobresaliente en la zona fría de los andes colombianos (Graves, 2006; Fondo Nacional del Fomento de la Papa [FNFP], 2016); se estima que en 2016 se cosecharon en Nariño 33800 ha de papa, que representaron el 21,0\% del área nacional (Red de Información y Comunicación del Sector Agropecuario de Colombia [AGRONET], 2019). En este departamento, para producir papa se utilizan dos tipos de labranza del suelo diferenciadas: la labranza comercial intensiva, que utiliza tractor e implementos pesados o implementos de tracción animal o manual con alto número de pases, y la siembra con surcado en wachado (surco o camellón), que utiliza solo una herramienta manual llamada pala de cute.

Estos tipos de labranza se practican en zonas ubicadas entre los 2700 a $3100 \mathrm{~m}$ de altitud, en suelos profundos, con pendientes pronunciadas de gran extensión, de baja densidad aparente, alta capacidad de retención de humedad, mediana estabilidad estructural y condiciones frágiles muy susceptibles a la erosión (Ordoñez, 2007). La labranza mecanizada intensiva, usualmente causa efectos significativos sobre la estabilidad estructural, al disminuir los organismos del suelo, romper terrones y agregados, y exponer la materia orgánica en la superficie, lo que afecta negativamente el volumen del suelo, su capacidad para retener y ceder agua a las plantas y la respuesta de los suelos y cultivos a la aplicación de fertilizantes (Lal, 1993).

La erosión en laderas y la compactación subsuperficial y pérdida de estructura en valles, son los factores principales de degradación del suelo en Colombia. Las fuerzas degradativas que actúan constantemente sobre el suelo son naturales, básicamente, y las inducidas de forma antrópica. Las naturales están representadas principalmente por la agresividad de las lluvias, producto de las manifestaciones de su intensidad (Colotti, 1999), y las antrópicas están relacionadas fundamentalmente con el uso indiscriminado, inoportuno e inapropiado de la práctica de labranza. El alto número de pases de implementos de labranza, incluidos los de tracción animal y humana, rompen o fraccionan terrones y agregados a microagregados que sellan el suelo y facilitan el arrastre (Amézquita, 1999).Algunos estudios con labranza mecanizada intensiva en la zona alta de Nariño, Colombia, reportaron pérdidas de suelo entre 80 a $100 \mathrm{t} \mathrm{ha}^{-1} \mathrm{año}^{-1}$, dependiendo de la pendiente; desestabilización física al reducirse el volumen del suelo en $28,0 \%$, infiltración en 71,0 \% y capacidad de aceptación de lluvias con niveles de 
escorrentía de 95,0 \%, ligeros cambios en la clase textural, disminución de contenidos de $\mathrm{Ca}, \mathrm{Mg}$ y materia orgánica del 59,0 \% (Volverás et al., 2007; Volverás \& Amézquita, 2009; Volverás et al., 2016). El sistema de siembra con surcado en wachado o wachu rozado, es de origen precolombino sostenido en la historia como resultado del legado cultural indígena (Valdez, 1987; Pumisacho \& Sherwood, 2002; Toapanta, 2004), quienes lo practican y conservan en algunas zonas paperas del departamento de Nariño y norte de Ecuador.

Se estima que en Nariño cubre $40 \%$ del área total en papa, donde se produce cerca del $40 \%$ de la papa y $35 \%$ de la leche del departamento, puesto que en el surcado en wachado se aplica un sistema mixto de producción donde el componente agrícola principal es la papa y el pecuario es el ganado de leche (Ordoñez, 2007; Yapa, 2013). Según la Organización para la Alimentación y la Agricultura (Food and Agriculture Organization, 2000; 2010), la Alianza Mundial para la Conservación y la Gestión de los Sistemas Ingeniosos del Patrimonio Agrícola Mundial (SIPAM), incluyó el surcado en wachado entre las prácticas o saberes ancestrales de cultivo en Perú, Colombia y Ecuador.

Parte de su fortaleza agroecológica radica en que el wachado para la siembra de papa, no destruye la cobertura del suelo y no utiliza maquinaria pesada, sino una herramienta manual llamada pala de cute. Después de dos o tres años con pastura, para la siembra de papa se aprovecha el cespedón de la capa superficial, para construir los surcos con la pala de cute en siembra y aporques. En el sistema wachado, en el entresurco, el productor pica con pala de cute el suelo hasta desmenuzarlo y lo deposita sobre el lomo del surco, lo cual genera dos efectos: a) al sacar el suelo se forma un canal que permanece durante todo el ciclo de cultivo y b) al depositarlo sobre el lomo del surco el suelo queda expuesto a la erosión.

La erosión del suelo es un proceso mecánico que requiere energía, gran parte de esta energía es suministrada por las gotas de lluvia; el peso y la gran velocidad de las gotas de hasta $32 \mathrm{~km} \mathrm{~h}^{-1}$ (Wischmeier \& Smith, 1978), impactan y también separan las partículas de suelo y destruyen los agregados superficiales; este material es transportado posteriormente por el flujo o escorrentía superficial, que para el caso de suelos en ladera la pendiente juega un papel importante (Lal, 1993), más aún si el surcado se realiza en el sentido de la pendiente, tal como se realiza tanto en labranza mecanizada como en wachado, que en la zona de Nariño está entre el 15 y 35 \% (Moore, 1979).

El sistema wachado es estratégico para generar empleo y alimentos, debido a que los productores destinan parte de la producción para autoconsumo (Campo et al., 2014). En lo literal wachado o wachu rozado (quechua) es un término agrícola que significa surco, camellón, wachay ensurcado o camellonado (Rozas, 2007); es un sistema de uso del suelo en laderas que constantemente rota uno o dos ciclos de cultivo la papa u otro tubérculo andino con tres a cinco años de pastura para la ganadería de leche. En la llanura del lago Titicaca en Perú y Bolivia, construyen camellones o surcos llamados waru-waru (quechua) o sucacollos (aymara), más anchos (4-5 m de ancho) que el wachado tradicional $(70$ a $90 \mathrm{~cm}$ ), para mitigar el efecto de las heladas, debido a que el agua que logran atrapar no permite la sublimación inversa cerca de los cultivos de papa (Ordoñez, 2007). Algunos campesinos de Perú y Bolivia, siguen cultivando en este tipo de camellones, como hicieron sus antepasados desde hace más de 2000 años; este ingenioso sistema de agricultura fue empleado en otros altiplanos húmedos de Ecuador como Quito, Cayambe y San Pablo (Stadel, 2005; Yapa, 2013).

$\mathrm{Si}$ el terreno es muy inclinado, los agricultores emplean surcos a favor de la pendiente llamados pasaq wachu y otros surcos inclinados para evitar la erosión del suelo; si la pendiente es mínima se utilizan los llamados kunka kunka, para evitar que el agua se encharque y se descompongan los tubérculos. Después de la cosecha de papa, para pasar a pastura ingresan los bovinos que consumen los residuos de cosecha y las malezas; después, con la pala de cute, aflojan el suelo y en el caso de Nariño siembran mezclas de leguminosas forrajeras y gramíneas (Volverás et al., 2014).

En comparación con la labranza mecanizada intensiva, Ordoñez (2007) y Volverás et al. (2014) en Nariño, Colombia, reportaron que el surcado en wachado a mediano y largo plazo, conserva la cobertura del suelo, disminuye la erosión, la compactación, mejora la porosidad total, la estabilidad estructural, la infiltración de agua y reduce la escorrentía. En Nariño (Bastidas, 2003) y en Ecuador (Ramos et al., 2002), se reportó que la siembra 
en wachado en papa disminuyó la compactación en la capa superficial para la segunda siembra; pero a mayor profundidad, se incrementó la compactación, humedad y densidad aparente.

En el surcado en wachu rozado en Ecuador se encontró que, aunque los surcos se construyen en el sentido de la pendiente, las raíces que brotan del cespedón de la pastura y crecen en los costados del surco, reducen la erosión del suelo por escurrimiento; además, mejora la actividad microbiana y crea un ambiente antagónico al gusano blanco y otras plagas del suelo (Pumisacho \& Sherwood, 2002). Además, la descomposición de la biomasa de pastura genera condiciones favorables para la toma de nutrientes y agua por el cultivo (Thompson \& Troeh, 2009).

En Ecuador, un estudio de caracterización de los sistemas de labranza, determinó que en wachado, fue superior el rendimiento de tubérculos (20\%), la disponibilidad de forraje (20\%), la carga animal (30\%), la diversidad de organismos del suelo (40 \%) y la relación costo/beneficio (30 \%) (Sánchez, 2009). Sin embargo, en Nariño, el sistema de surcado en wachado presenta varias limitantes técnicas en algunas labores; para el uso de la pastura, utilizan alta carga animal con largos períodos de ocupación que afecta la disponibilidad y persistencia de la pastura y compacta los suelos. Debido a la importancia del surcado en wachado como sistema de uso del suelo en laderas andinas, el objetivo del presente trabajo fue determinar el efecto de la inclinación del surco en wachado para la siembra de papa sobre la pérdida de suelo en la zona alto andina de Nariño.

\section{Materiales y métodos}

\section{Localización}

Esta investigación se realizó en la región andina, eco región sabana y montaña, en la zona de vida de bosque húmedo Montano (bh-M) (Holdridge, 1987), en el departamento de Nariño, municipios de Pasto, vereda Río Bobo (N 0106'36,7”, W 77¹7'57,4”), suelo Typic Fulvudands; Potosí, vereda Villa Nueva (N 0048’38,8”, W 77³0'54,01'), suelo Typic Hapludands y Pupiales, vereda Imbula (N 0057’20,4”, W 77³7'10,7’), suelo Pachic Melanudands, ubicados dentro la subregión Nudo de los Pastos (Instituto Geográfico Agustín Codazzi, 2004). En general son suelos profundos, livianos y de fertilidad media a alta. Algunos valores de referencia de indicadores de fertilidad química de los suelos de las localidades de estudio y los niveles de precipitación media mensual de la subregión Nudo de los Pastos se presentan en los Cuadros 1 y 2.

\section{Surcado con el sistema de wachado}

El cultivo de papa (Solanum tuberosum) en wachado consiste en el mínimo laboreo del suelo en la siembra y en la utilización del cespedón (porción superficial de césped y suelo, Figura 1b) de la pastura, para lo cual se cortan con una herramienta manual denominada pala de cute (la pala de cute es una pala a la cual se adiciona con soldadura una extensión de hierro y un mango de madera curvo, Figura 1a) que corta y voltea cespedones hacia adentro para formar el surco denominado wachu.

La siembra de papa en wachado, se realiza en el sentido de pendientes pronunciadas y de gran extensión, y para las labores de tapado de la semilla después de siembra y aporque (acumular suelo en la base del tallo), con la pala de cute se afloja el suelo del entresurco disgregándolo y ubicándolo en el lomo del surco que genera una zanja profunda (Figura 2 y 3); estas labores, favorecen la escorrentía, la disminución de la capacidad del suelo para captar almacenar y ceder agua y las condiciones para pérdida de suelo.

Las fincas se seleccionaron en los municipios donde la siembra con surcado en wachado es el sistema predominante, que estuvieron en pastura durante tres años. A principios de mayo de 2014 se realizó el pastoreo y luego se trazaron los tratamientos de surcado. Debido a la compleja topografía de la zona y la disponibilidad de 
Cuadro 1. Resultados análisis químico de suelo a dos profundidades para las localidades de estudio, Nariño, Colombia. 2014.

Table 1. Soil chemical analysis results at two depths for the study locations, Nariño, Colombia. 2014.

\begin{tabular}{|c|c|c|c|c|c|c|c|}
\hline \multirow[b]{2}{*}{ Parámetro } & \multirow[b]{2}{*}{ Unidades } & \multicolumn{2}{|c|}{ Pasto } & \multicolumn{2}{|c|}{ Pupiales } & \multicolumn{2}{|c|}{ Potosí } \\
\hline & & $0-20 \mathrm{~cm}$ & $21-40 \mathrm{~cm}$ & $0-20 \mathrm{~cm}$ & $21-40 \mathrm{~cm}$ & $0-20 \mathrm{~cm}$ & $21-40 \mathrm{~cm}$ \\
\hline MO & $\%$ & 19,65 & 20,83 & 14,44 & 13,07 & 8,45 & 4,67 \\
\hline $\mathrm{pH}$ & & 5,06 & 4,94 & 4,93 & 5,14 & 5,29 & 5,17 \\
\hline CE & $\mathrm{ds} \mathrm{m}^{-1}$ & 0,22 & 0,28 & 0,15 & 0,14 & 0,13 & 0,08 \\
\hline $\mathrm{Ca}$ & $\mathrm{cmol} \mathrm{kg}^{-1}$ & 0,82 & 0,63 & 1,48 & 1,53 & 2,65 & 1,15 \\
\hline $\mathrm{Mg}$ & $\mathrm{cmol} \mathrm{kg}^{-1}$ & 0,27 & 0,19 & 0,21 & 0,24 & 0,25 & 0,15 \\
\hline $\mathrm{K}$ & $\mathrm{cmol} \mathrm{kg}^{-1}$ & 0,78 & 0,50 & 0,28 & 0,20 & 0,56 & 0,33 \\
\hline $\mathrm{Na}$ & $\mathrm{cmol} \mathrm{kg}^{-1}$ & 0,08 & 0,08 & 0,05 & 0,07 & 0,08 & 0,07 \\
\hline CICE & $\mathrm{cmol} \mathrm{kg}^{-1}$ & 4,45 & 3,70 & 3,85 & 3,90 & 4,38 & 2,47 \\
\hline $\mathrm{Al}+\mathrm{H}$ & $\mathrm{cmol} \mathrm{kg}^{-1}$ & 2,50 & 2,30 & 1,83 & 1,86 & 0,85 & 0,77 \\
\hline $\mathrm{Al}$ & $\mathrm{cmol} \mathrm{kg}^{-1}$ & 2,28 & 2,19 & 1,74 & 1,82 & 0,52 & 0,65 \\
\hline Sat Al & $\%$ & 51,40 & 59,20 & 45,30 & 46,60 & 11,90 & 26,20 \\
\hline $\mathrm{P}$ & $\mathrm{mg} \mathrm{kg}^{-1}$ & 29,20 & 2,00 & 110,30 & 36,60 & 43,10 & 8,80 \\
\hline S & $\mathrm{mg} \mathrm{kg}^{-1}$ & 7,70 & 8,30 & 11,10 & 13,90 & 6,60 & 11,30 \\
\hline B & $\mathrm{mg} \mathrm{kg}^{-1}$ & 0,20 & 0,20 & 0,25 & 0,15 & 0,20 & 0,18 \\
\hline $\mathrm{Cu}$ & $\mathrm{mg} \mathrm{kg}^{-1}$ & 3,00 & 2,60 & 1,30 & 1,50 & 2,80 & 3,00 \\
\hline $\mathrm{Fe}$ & $\mathrm{mg} \mathrm{kg}^{-1}$ & 414,00 & 561,00 & 436,00 & 815,00 & 287,00 & 215,00 \\
\hline Mo & $\mathrm{mg} \mathrm{kg}^{-1}$ & 31,30 & 18,60 & 8,00 & 4,80 & 17,90 & 18,00 \\
\hline $\mathrm{Zn}$ & $\mathrm{mg} \mathrm{kg}^{-1}$ & 4,50 & 3,10 & 6,50 & 4,20 & 2,90 & 1,60 \\
\hline
\end{tabular}

Cuadro 2. Precipitación y evaporación (mm) media mensual de la subregión Nudo de los Pastos, Nariño, Colombia. 2014.

Table 2. Average monthly precipitation and evaporation (mm) of the subregion Nudo de los Pastos, Nariño, Colombia. 2014.

\begin{tabular}{lccccccccc}
\hline Localidad y parámetro & Abr & May & Jun & Jul & Ago & Sep & Oct & Nov & Total, período \\
\hline Pasto precipitación & 135,0 & 98,9 & 52,0 & 94,8 & 14,2 & 29,0 & 109,5 & 84,4 & 617,8 \\
Pasto evaporación & 71,4 & 81,4 & 68,3 & 76,5 & 107,2 & 81,7 & 90,4 & 96,5 & 673,4 \\
Potosí lluvias & 90,6 & 198,2 & 19,1 & 38,0 & 30,6 & 19,5 & 136,0 & 87,3 & 619,3 \\
Potosí evaporación & 65,5 & 80,0 & 80,4 & 88,8 & 97,2 & 84,5 & 106,0 & 93,4 & 695,8 \\
Pupiales lluvias & 67,0 & 133,3 & 19,2 & 50,6 & 18,8 & 22,0 & 66,5 & 139,4 & 516,8 \\
Pupiales evaporación & 76,6 & 73,8 & 70,4 & 60,8 & 86,0 & 79,5 & 91,5 & 69,8 & 608,4 \\
\hline
\end{tabular}

Fuente / Source: Instituto de Hidrología, Meteorología y Estudios Ambientales (2014).

fincas, no fue posible seleccionar lotes exactamente similares. Se utilizó semilla certificada de papa de la variedad Diacol Capiro (Solanum tuberosum sp. andigena) que en Colombia es la principal variedad para consumo en fresco y proceso industrial.

Los tratamientos de inclinación del surco se definieron en acuerdo con los productores quienes, no inclinaban demasiado el surco por la dificultad para operar la pala de cute en pendientes superiores al $30 \%$ y también se consideró el trabajo de Ordoñez (2007) quien, en un trabajo en Nariño, Colombia, realizó surcado en wachado a 

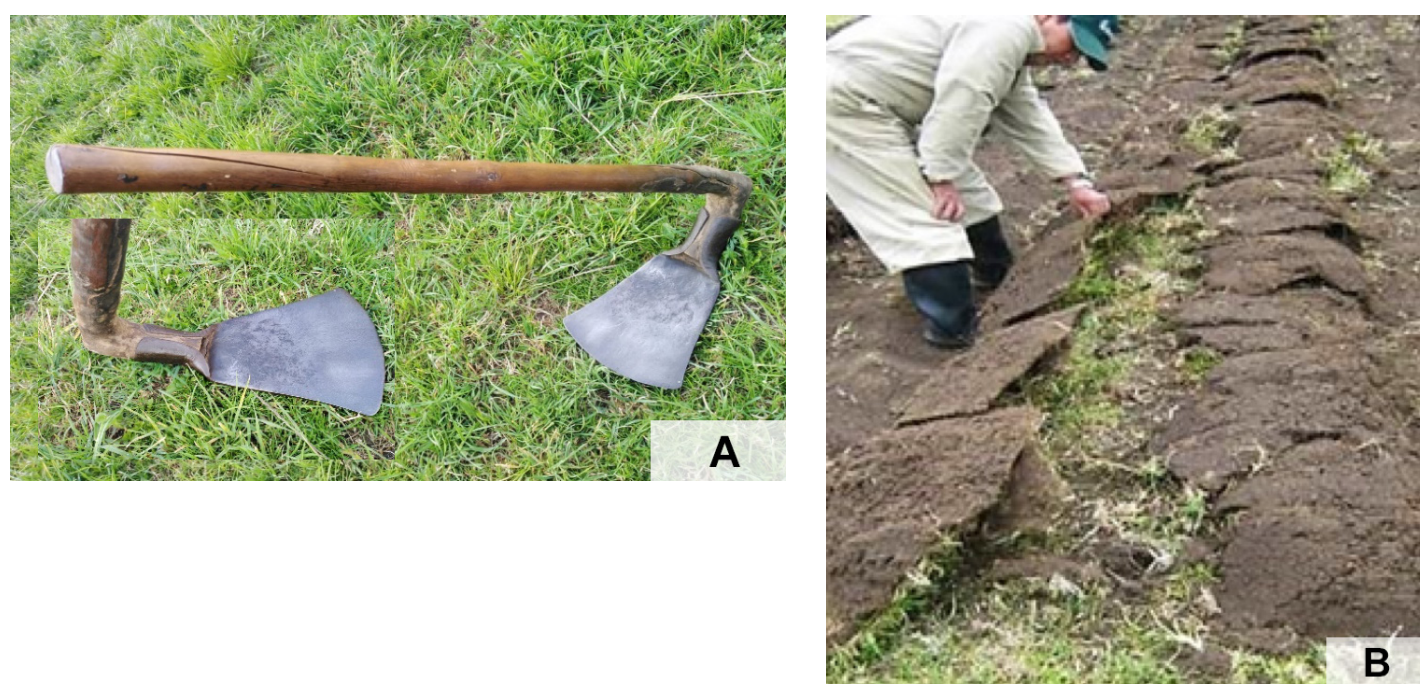

Figura 1. (a). La herramienta manual llamada "pala de cute", para las labores culturales en wachado. (b). Surcos construidos con cespedones de la pastura para la siembra de la papa en wachado (surco o camellón) en laderas andinas de Nariño, Colombia. 2014.

Figure 1. (a). The manual tool called "cute shovel" for cultural work in wachado. (b). Furrows built with grasses for potato planting in wachado (furrow or ridge) on Andean slopes of Nariño, Colombia. 2014.

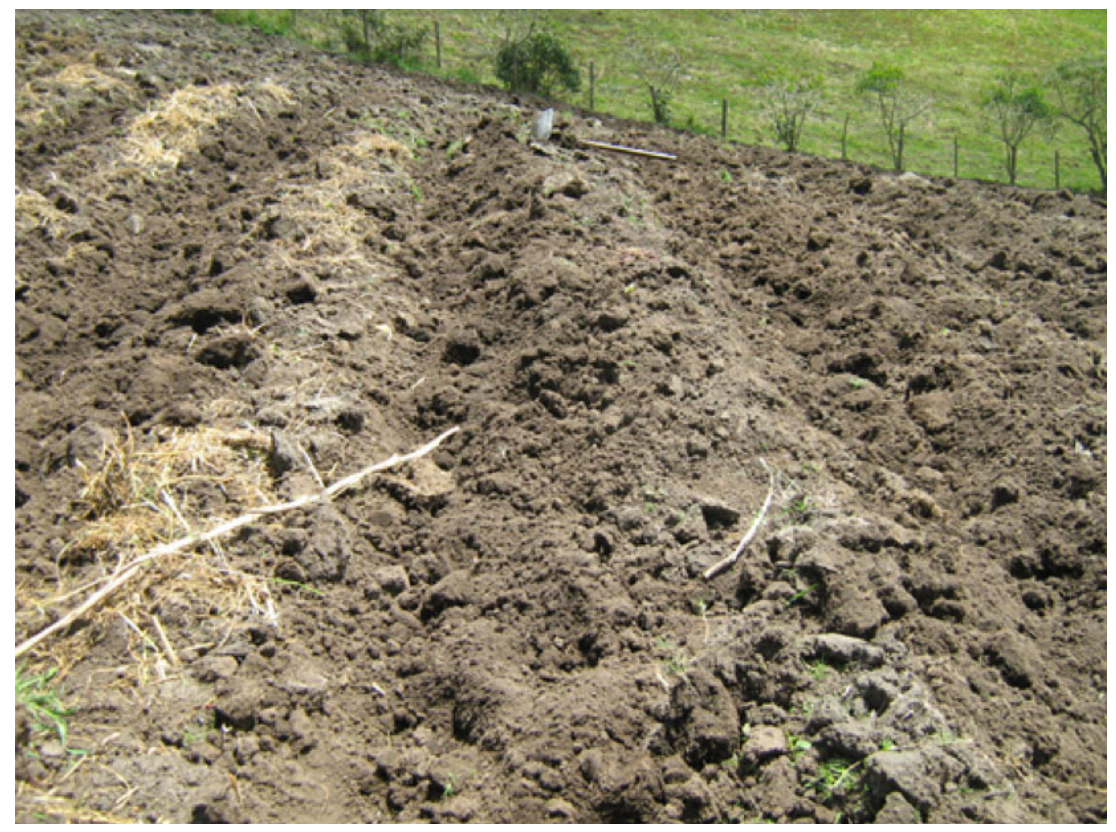

Figura 2. Suelo disturbado para la labor de tapado de la semilla en el sistema de siembra de papa (Solanum tuberosum) en wachado (surco o camellón), en laderas andinas del departamento de Nariño, Colombia. 2014.

Figure 2. Disturbed soil for the work of covering the seed in the system of potato (Solanum tuberosum) sowing in wachado (furrow or ridge) in Andean slopes of the department of Nariño, Colombia. 2014. 


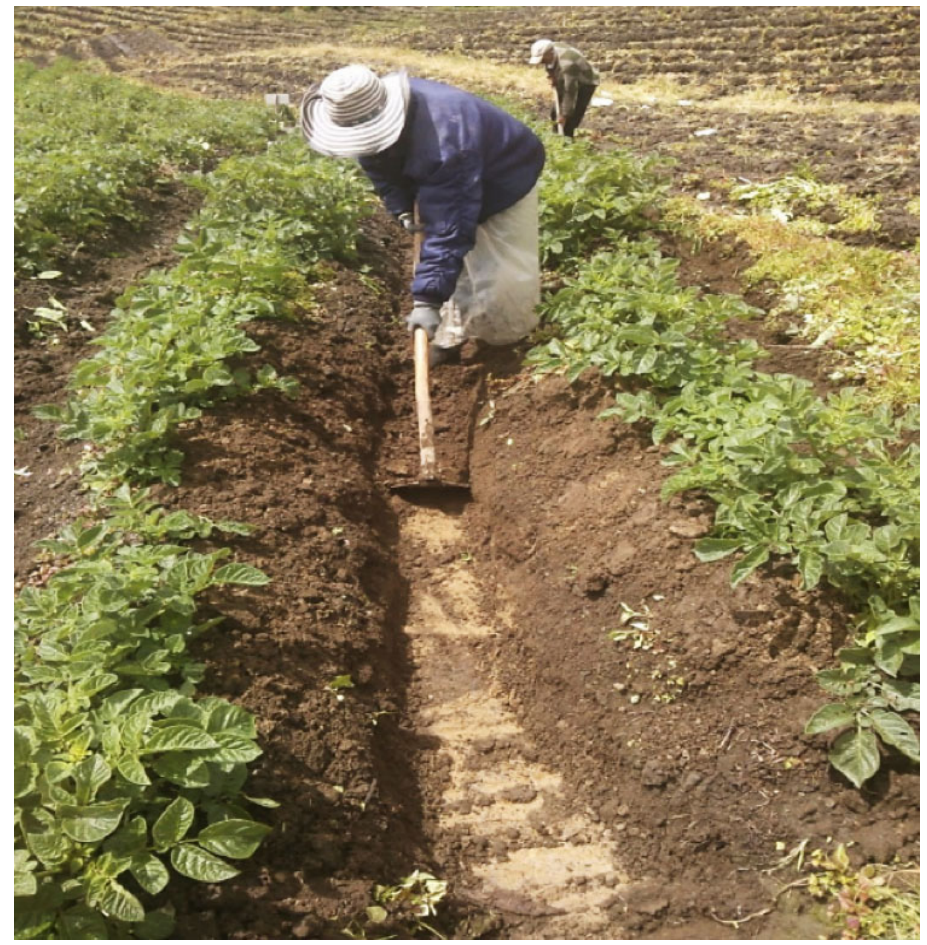

Figura 3. Remoción del suelo en el entre surco para realizar el primer aporte que favorece las pérdidas de suelo por escorrentía en las laderas andinas del departamento de Nariño, Colombia. 2014.

Figure 3. Removal of the soil in the interfurrow to make the first hilling that favors soil losses due to runoff on the Andean slopes of the Nariño department, Colombia. 2014.

$30^{\circ}$ y $45^{\circ}$, pero encontraron dificultades para realizar el surcado en pendientes mayores al $30 \%$ y afectación en la producción de papa por acumulación de humedad en los bordes del surco.

\section{Diseño experimental}

Se utilizó un diseño bloques completos al azar con arreglo factorial (localidad, época de muestreo e inclinación del surco). En cada localidad el experimento tuvo tres bloques o repeticiones. La repetición o bloque estuvo compuesta por cuatro niveles de inclinación del surco, para un total de doce parcelas o unidades experimentales en cada localidad; la unidad experimental constó de seis surcos de $13 \mathrm{~m}$ de largo distanciados a 1,4 m (109,2 m²); las parcelas o unidades experimentales se aislaron con láminas de zinc y en la parte de abajo se construyeron canaletas recubiertas con plástico para recoger el suelo erosionado. Los niveles o grados de inclinación del surco fueron: wachado a $90^{\circ}, 70^{\circ}$ y $60^{\circ}$ sobre la horizontal, y labranza comercial, esta última, consiste en labores con maquinaria agrícola, labranza con dos a tres pases de arado de discos y dos a tres pases con rastrillo liviano y surcado que puede ser con surcadora mecánica o con tracción animal.

Debido a situaciones de logística y de condiciones climáticas de cada localidad (Pasto, Potosí y Pupiales), las siembras de cada lote experimental no fueron simultáneas, pero la evaluación de la pérdida de suelo en cada localidad se realizó en las épocas de mayor laboreo dentro del ciclo del cultivo como son siembra, aporque uno, aporque dos, controles de malezas y cosecha, épocas que difirieron en cada localidad en razón del clima, manejo agronómico y desarrollo del cultivo. 
Para el trazado de los tratamientos de los surcos, en la base de los lotes se trazó una línea horizontal sobre la cual se midieron los ángulos de las inclinaciones. La información se analizó mediante el uso del paquete estadístico SAS (SAS Institute Inc., version 9.4); se realizó un procedimiento GLM y un análisis de varianza combinado; asimismo, se hicieron las pruebas de normalidad correspondientes y las pruebas Tukey (HSD) para comparación de medias en los casos en que se detectaran diferencias estadísticas significativas a un nivel de $\alpha=0,01$.

\section{Variables evaluadas}

Pérdida de suelo

Terminada la labor agronómica en cada una de las seis épocas de evaluación, se procedió a recoger y pesar el suelo que cayó en las canaletas, y en las cuales también se determinó humedad gravimétrica para expresar la información como peso de suelo seco.

\section{Rendimiento de papa}

En la parte central de cada parcela se cosechó un área de 3,0 m por 2,6 m $\left(7,8 \mathrm{~m}^{2}\right)$ y se tomó el peso fresco $(\mathrm{kg})$ de cada uno de los cuatro tamaños del tubérculo de papa, que según la norma NTC 341 (Instituto Colombiano de Normas Técnicas y Certificación, 1996) son, tipo cero (diámetro mayor de $90 \mathrm{~mm}$ ), tipo 1 (de 65 a $90 \mathrm{~mm}$ ), tipo 2 (de 45 a $64 \mathrm{~mm})$ y tipo 3 (30 a $44 \mathrm{~mm})$.

\section{Resultados}

Hubo efecto significativo $(\mathrm{p}<0,001)$ de la localidad, el nivel de inclinación del surco, las épocas de evaluación

y de las interacciones localidad*épocas y épocas*nivel de inclinación del surco, sobre la pérdida de suelo; debido a un problema externo al desarrollo del trabajo, no fue posible tomar la información de rendimiento de papa en la localidad de Pupiales, razón por la cual solo se presenta lo obtenido en Pasto y Potosí.

\section{Pérdida de suelo por efecto de la localidad}

Las localidades de Pupiales y Potosí presentaron la mayor pérdida de suelo con 65,84 y 64,98 t ha-1, respectivamente. La pérdida en Pasto fue $34 \%$ menor con 43,49 $\mathrm{t} \mathrm{ha}^{-1}$ (Figura 4).

\section{Pérdida de suelo por efecto del nivel de inclinación del surco}

La labranza comercial y el wachado a $90^{\circ}$, presentaron mayor pérdida de suelo con 69,28 y $63,5 \mathrm{t} \mathrm{ha}^{-1}$ respectivamente; al pasar a wachado a 60 y $70^{\circ}$, la pérdida de suelo disminuyó $35 \%$ y $23 \%$, respectivamente, frente a labranza comercial. La pérdida de suelo en wachado a $70^{\circ}$ fue de $53,5 \mathrm{t} \mathrm{ha}^{-1}$, un $23 \%$ y $16 \%$ menor frente a labranza comercial y wachado a $90^{\circ}$, respectivamente (Figura 5).

\section{Pérdida de suelo por efecto de la época de evaluación}

La pérdida de suelo por época fue mayor y similar en cosecha $\left(15,82 \mathrm{t} \mathrm{ha}^{-1}\right)$ y aporque uno $\left(15,28 \mathrm{t} \mathrm{ha}^{-1}\right)$, seguida en su orden por siembra $\left(14,04 \mathrm{t} \mathrm{ha}^{-1}\right)$, aporque dos $\left(11,84 \mathrm{t} \mathrm{ha}^{-1}\right)$ y floración y madurez con 0,83 y $0,26 \mathrm{t}$ 


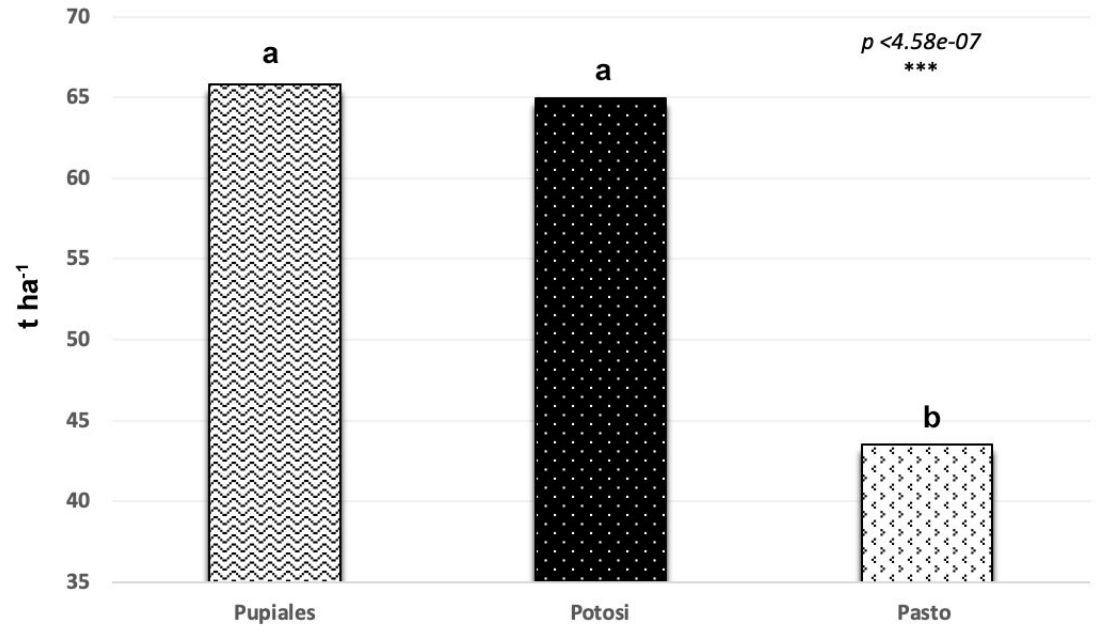

Figura 4. Pérdida de suelo $\left(\mathrm{t} \mathrm{ha}^{-1}\right)$ por localidad por causa de erosión hídrica en un ciclo de cultivo de papa (Solanum tuberosum) en wachado (surco o camellón). Departamento de Nariño, Colombia. 2014.

Figure 4. Soil loss $\left(\mathrm{t} \mathrm{ha}^{-1}\right)$ by locality due to water erosion in a potato (Solanum tuberosum) crop cycle in wachado (furrow or ridge). Nariño department, Colombia. 2014.

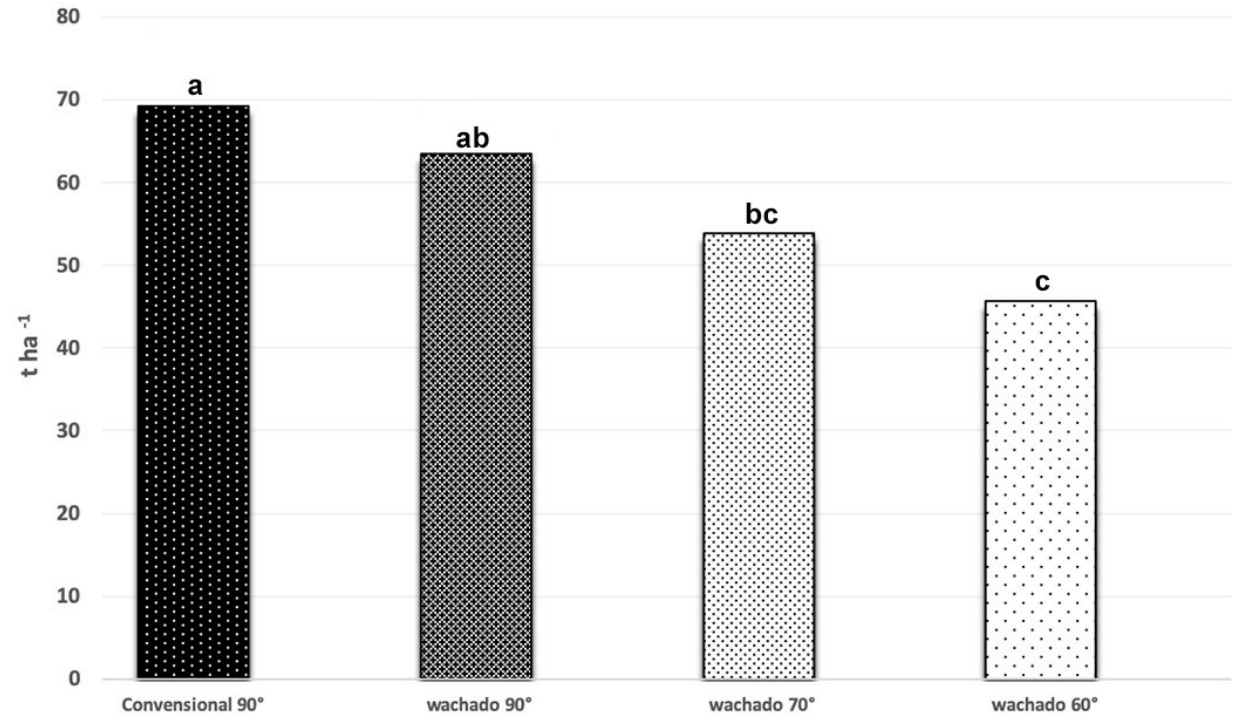

Figura 5. Pérdida de suelo ( $\mathrm{t} \mathrm{ha}^{-1}$ ) según el nivel de inclinación del surco en wachado (surco o camellón), en un ciclo de cultivo de papa (Solanum tuberosum), departamento de Nariño, Colombia. 2014.

Figure 5. Soil loss $\left(\mathrm{t} \mathrm{ha}^{-1}\right)$ according to the level of the furrow inclination in wachado (furrow or ridge), in a potato (Solanum tuberosum) cultivation cycle, department of Nariño, Colombia, 2014.

ha $^{-1}$, respectivamente. Las épocas de mayor laboreo coincidieron con las actividades donde el agricultor utilizó la pala de cute para remover el suelo en el surco y en el entresurco (Figura 6). 


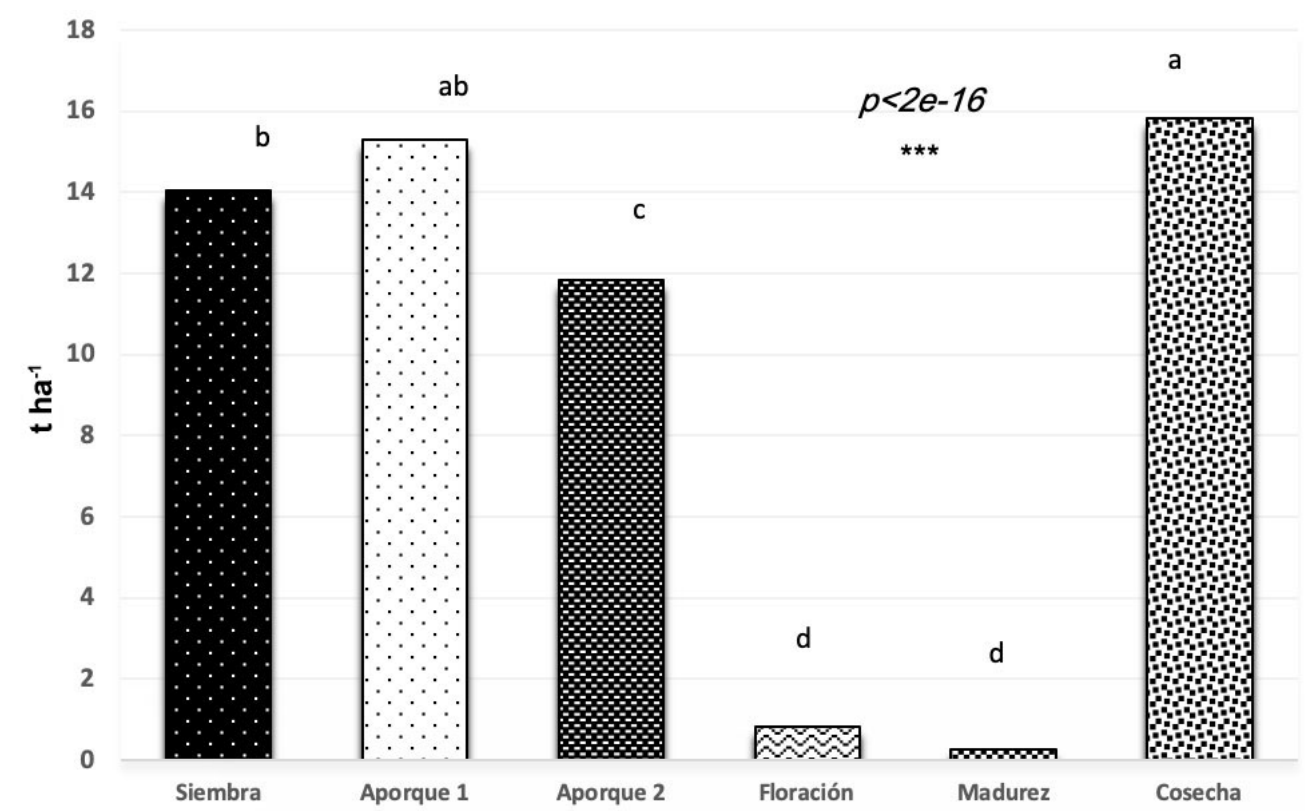

Figura 6. Pérdida de suelo $\left(\mathrm{t} \mathrm{ha}^{-1}\right)$ por efecto de la inclinación del surco en wachado (surco o camellón), en cada una de las épocas de evaluación en un ciclo de cultivo de papa (Solanum tuberosum), departamento de Nariño, Colombia. 2014.

Figure 6. Soil loss $\left(\mathrm{t} \mathrm{ha}^{-1}\right)$ due to the effect of the furrow inclination in wachado (furrow or ridge), in each of the evaluation periods in a potato (Solanum tuberosum) cultivation cycle, department of Nariño, Colombia, 2014.

\section{Pérdida de suelo por efecto de la interacción localidad por épocas de evaluación}

Las menores pérdidas de suelo fueron entre 0,38 y $1,27\left(\mathrm{t} \mathrm{ha}^{-1}\right)$ y se presentaron en las épocas de floración y madurez en las tres localidades, sin diferencias estadísticas entre estas; asimismo, las mayores pérdidas se presentaron en cosecha, aporque uno, siembra y aporque dos en las tres localidades (Cuadro 3). La pérdida de suelo de las épocas varió con la localidad; en siembra y cosecha, la pérdida de suelo en Pasto fue en promedio, $46 \%$ y $73 \%$ menor en comparación con las otras dos localidades, pero en aporque dos fue $18 \%$ mayor. En general, en

Cuadro 3. Pérdida de suelo $\left(\mathrm{t} \mathrm{ha}^{-1}\right)$ en tres localidades y seis épocas de evaluación, en papa (Solanum tuberosum) cultivada en wachado (surco o camellón). Nariño, Colombia 2014.

Table 3. Soil loss $\left(\mathrm{t} \mathrm{ha}^{-1}\right)$ in three locations and six evaluation periods, in potato (Solanum tuberosum) cultivation in wachado (furrow or ridge). Nariño, Colombia 2014.

\begin{tabular}{lcccccc}
\hline \multirow{2}{*}{ Localidades } & \multicolumn{9}{c}{ Erosión por épocas } \\
\cline { 2 - 7 } & Siembra & Aporque uno & Aporque dos & Floración & Madurez & Cosecha \\
\hline Pasto río Bobo & $9,01 \mathrm{~g}$ & $16,90 \mathrm{~cd}$ & $13,66 \mathrm{de}$ & $0,38 \mathrm{~h}$ & $0,34 \mathrm{~h}$ & $3,21 \mathrm{~h}$ \\
Potosí Villa Nueva & $12,29 \mathrm{ef}$ & $17,42 \mathrm{c}$ & $10,13 \mathrm{efg}$ & $0,86 \mathrm{~h}$ & $0,08 \mathrm{~h}$ & $24,20 \mathrm{a}$ \\
Pupiales Imbula & $20,84 \mathrm{~b}$ & $11,54 \mathrm{efg}$ & $11,75 \mathrm{efg}$ & $1,27 \mathrm{~h}$ & $0,37 \mathrm{~h}$ & $20,06 \mathrm{bc}$ \\
\hline
\end{tabular}

Medias con la misma letra no son significativamente diferentes $(\mathrm{p}<0,01)$ / Means with the same letter were not significantly different $(\mathrm{p}<0.01)$. 
las tres localidades las menores pérdidas coincidieron cuando no se usó la pala de cute y solo se hicieron controles sanitarios con bomba de espalda y machete para cortar malezas en el borde del surco (Cuadro 3).

\section{Pédida de suelo según inclinación del surco y época de evaluación}

Las mayores pérdidas de suelo se presentaron en los surcos a $90^{\circ}$ de inclinación en la época de cosecha, siembra y aporque uno, con valores que oscilaron entre 20,45 y 16,65 $\mathrm{t} \mathrm{ha}^{-1}$. En todos los niveles de inclinación del surco, las menores pérdidas se presentaron en las épocas de floración y madurez con valores que variaron entre 0,91 y 0,16 t ha $^{-1}$ (Cuadro 4).

Cuadro 4. Pérdida de suelo $\left(\mathrm{t} \mathrm{ha}^{-1}\right)$ por época de evaluación en el cultivo de papa (Solanum tuberosum) cultivada en wachado (surco o camellón), y bajo diferentes niveles de inclinación del surco, departamento Nariño, Colombia. 2014.

Table 4. Soil loss $\left(\mathrm{t} \mathrm{ha}^{-1}\right)$ by season of assessment in potato (Solanum tuberosum) cultivation in wachado (furrow or ridge), and under different levels of slope of the furrow, Nariño department, Colombia. 2014.

\begin{tabular}{lcccccc}
\hline & Siembra & Aporque uno & Aporque dos & Floración & Madurez & Cosecha \\
\hline Wachado $90^{\circ}$ & $16,65 \mathrm{ab}$ & $16,47 \mathrm{~b}$ & $12,43 \mathrm{cde}$ & $0,82 \mathrm{f}$ & $0,37 \mathrm{f}$ & $16,73 \mathrm{ab}$ \\
Wachado $70^{\circ}$ & $12,16 \mathrm{cde}$ & $14,14 \mathrm{bcd}$ & $11,12 \mathrm{de}$ & $0,76 \mathrm{f}$ & $0,17 \mathrm{f}$ & $15,50 \mathrm{bc}$ \\
Wachado $60^{\circ}$ & $10,98 \mathrm{de}$ & $13,57 \mathrm{bcd}$ & $9,52 \mathrm{e}$ & $0,91 \mathrm{f}$ & $0,16 \mathrm{f}$ & $10,60 \mathrm{de}$ \\
Comercial $90^{\circ}$ & $16,38 \mathrm{~b}$ & $16,95 \mathrm{ab}$ & $14,30 \mathrm{bcd}$ & $0,84 \mathrm{f}$ & $0,33 \mathrm{f}$ & $20,45 \mathrm{a}$ \\
\hline
\end{tabular}

Medias con la misma letra no son significativamente diferentes $(\mathrm{p}<0,01)$ / Means with the same letter were not significantly different $(\mathrm{p}<0.01)$.

Con excepción de la pérdida de suelo en floración, en todas las épocas, la pérdida de suelo disminuyó al reducir la inclinación del surco; en cosecha, la pérdida de suelo del wachado a $60^{\circ}$ se redujo $48 \%$ y $37 \%$ frente a labranza comercial y wachado a $90^{\circ}$. En siembra, la reducción fue del $33 \%$ y $35 \%$ (Cuadro 4 ).

\section{Pérdida de suelo por localidad en los niveles de inclinación del surco durante el ciclo de cultivo}

La pérdida de suelo durante los cinco meses de cultivo fue afectada en forma significativa $(\mathrm{p}<0,001)$ por los factores nivel de inclinación de surcado y localidad. Las mayores pérdidas totales de suelo se alcanzaron en surcos en el sentido de la pendiente $\left(90^{\circ}\right)$ y en las localidades de Potosí y Pupiales. En labranza comercial la pérdida fue 74,37 y 78,69 t ha ${ }^{-1}$ y para wachado a $90^{\circ} 69,08$ y 75,83 tha ${ }^{-1}$ para Potosí y Pupiales, respectivamente; en este nivel de inclinación, la pérdida de suelo se redujo a $54,81 \mathrm{t} \mathrm{ha}^{-1} \mathrm{en}$ labranza comercial y $45,62 \mathrm{t} \mathrm{ha}^{-1}$ en wachado a $90^{\circ}$ en Pasto (Cuadro 5).

$\mathrm{Al}$ pasar a wachado a $70^{\circ}$ y $60^{\circ}$, la pérdida de suelo siguió siendo mayor en Potosí y Pupiales. En la pérdida de suelo durante todo el ciclo de cultivo, se encontró que esta disminuyó al reducir el nivel de inclinación del surcado. Frente a la labranza comercial a $90^{\circ}$, al pasar a surcado a $60^{\circ}$, la pérdida de suelo se redujo $41 \%, 31 \%$ y $32 \%$ en Pasto, Potosí y Pupiales, respectivamente. En su orden, al pasar a surcado a 70, la reducción fue del $25 \%, 12 \%$ y 29,6 \%.

La pérdida de suelo fue también menor en wachado a $90^{\circ}$ frente a la labranza comercial a $90^{\circ}$; para las localidades de Pasto, Potosí y Pupiales, la reducción fue del 16,7 \%, 7,0 \% y 3,6 \%, respectivamente. Aunque esta reducción fue menor en comparación con los otros niveles de inclinación del surcado, es importante y en el largo plazo, representa menor impacto en el proceso de degradación del suelo en laderas andinas (Cuadro 5). 
Cuadro 5. Pérdida total de suelo ( $\mathrm{t}$ ha ${ }^{-1}$ ) durante un ciclo de cultivo de papa (Solanum tuberosum) en wachado (surco o camellón), en tres localidades de Nariño, Colombia. 2014.

Table 5. Total loss of soil $\left(\mathrm{t} \mathrm{ha}^{-1}\right)$ during a crop cycle of potato (Solanum tuberosum) in wachado (furrow or ridge), in three locations in Nariño, Colombia 2014.

\begin{tabular}{lcccc}
\hline & \multicolumn{4}{c}{ Tratamiento surcado } \\
\cline { 2 - 5 } Localidades & ${\text { Wachado } \mathbf{9 0}^{\circ}}$ & ${\text { Wachado } \mathbf{7 0}^{\circ}}$ & ${\text { Wachado } \mathbf{6 0}^{\circ}}$ & Convencional 90 $^{\circ}$ \\
\hline Pasto río Bobo & $45,62 \mathrm{de}$ & $41,01 \mathrm{e}$ & $32,53 \mathrm{e}$ & 54,81 bcde \\
Potosí Villa Nueva & $69,08 \mathrm{abc}$ & $65,23 \mathrm{abcd}$ & $51,28 \mathrm{cde}$ & $74,37 \mathrm{abc}$ \\
Pupiales Imbula & $75,83 \mathrm{ab}$ & $55,39 \mathrm{bcde}$ & $53,46 \mathrm{bcde}$ & $78,69 \mathrm{a}$ \\
\hline
\end{tabular}

Medias con la misma letra no son significativamente diferentes $(\mathrm{p}<0,01) /$ Means with the same letter are not significantly different $(\mathrm{p}<0.01)$.

\section{Rendimiento de papa}

Tanto en Pasto como en Potosí no se encontró efecto de los tratamientos sobre el tamaño de los tubérculos de papa y rendimiento total. El rendimiento promedio en las dos localidades estuvo por arriba de $40 \mathrm{t} \mathrm{ha}^{-1}$, el cual fue consistente con los rendimientos en la zona de wachado y fue $55 \%$ superior al promedio regional $\left(18,0 \mathrm{t} \mathrm{ha}^{-1}\right)$ con labranza mecanizada intensiva (Cuadro 6). En Pasto, en todos los tratamientos cerca del $90 \%$ fue de tamaño de tubérculo comercial (tamaños cero, uno y dos) y en Potosí cerca del 94\%.

Cuadro 6. Rendimiento de tubérculos de papa (Solanum tuberosum) ( $\mathrm{t} \mathrm{ha}^{-1}$ ) por tamaños y total en wachado (surco o camellón), en dos localidades de Nariño, Colombia. 2014.

Table 6. Yield of potato (Solanum tuberosum) tubers $\left(\mathrm{t} \mathrm{ha}^{-1}\right)$ by size and total in wachado (furrow or ridge) in two localities of Nariño, Colombia. 2014.

\begin{tabular}{|c|c|c|c|c|c|c|}
\hline \multirow{2}{*}{ Localidades } & \multirow{2}{*}{$\begin{array}{c}\text { Tratamientos } \\
\text { labranza }\end{array}$} & \multicolumn{4}{|c|}{ Tamaño tubérculos* } & \multirow{2}{*}{$\begin{array}{c}\text { Rendimiento } \\
\text { total }\end{array}$} \\
\hline & & 0 & 1 & 2 & 3 & \\
\hline \multirow{4}{*}{ Pasto río Bobo } & wachado $90^{\circ}$ & 5,17 & 24,04 & 11,56 & 3,80 & 44,57 \\
\hline & wachado $70^{\circ}$ & 4,27 & 21,21 & 11,07 & 3,56 & 40,12 \\
\hline & wachado $60^{\circ}$ & 2,56 & 23,10 & 11,90 & 4,02 & 41,58 \\
\hline & Labranza comercial & 6,15 & 24,46 & 8,50 & 2,79 & 41,90 \\
\hline Promedio tamaños & & 4,54 & 23,20 & 10,76 & 3,54 & 42,04 \\
\hline \multirow{4}{*}{ Potosí Villa Nueva } & wachado $90^{\circ}$ & 2,28 & 29,23 & 13,85 & 2,44 & 47,79 \\
\hline & wachado $70^{\circ}$ & 0,89 & 27,50 & 11,82 & 2,50 & 42,71 \\
\hline & wachado $60^{\circ}$ & 3,37 & 26,46 & 11,83 & 2,04 & 43,69 \\
\hline & Labranza comercial & 3,05 & 28,42 & 11,82 & 2,27 & 45,56 \\
\hline Promedio tamaños & & 2,40 & 27,90 & 12,33 & 2,31 & 44,94 \\
\hline
\end{tabular}

*Tamaño de tubérculos: 0: diámetro mayor a 90 mm; 1: entre 65 y 90 mm; 2: entre 45 y 64 mm; 3: entre 30 y 44 mm / Tuber size: 0: diameter greater than $90 \mathrm{~mm}$; 1: between 65 and $90 \mathrm{~mm}$; : between 45 and $64 \mathrm{~mm}$; 3: between 30 and $44 \mathrm{~mm}$. 


\section{Discusión}

La mayor pérdida de suelo se obtuvo en labranza comercial intensiva con el surcado con en el sentido de la pendiente $\left(69,28 \mathrm{t} \mathrm{ha}^{-1}\right)$; cuando se pasó a wachado, donde no se utilizó maquinaria ni implementos pesados, la pérdida de suelo disminuyó inclusive a $90^{\circ}\left(63,5 \mathrm{t} \mathrm{ha}^{-1}\right)$, y gradualmente al reducir el nivel de inclinación del surcado (Figura 5).

Los resultados de la pérdida acumulada de suelo en cada localidad, se relacionaron con el manejo del componente de pastura. En la localidad de Pasto, con una pérdida de 43,49 t ha ${ }^{-1}$, la pastura estuvo compuesta por azul orchoro (Dactylis glomerata L), kikuyo (Cenchrus clandestinus Hochst. ex Chiov. Morrone) y trébol (Trifolium pratense), especies con mayor adaptación, lo cual les permite mayor persistencia en el tiempo, a diferencia de las otras dos localidades con una pérdida promedio de $65,41 \mathrm{t} \mathrm{ha}^{-1}$, donde el productor utilizaba raigrás (Lolium sp.), el cual es menos persistente en el tiempo y requiere de dos a tres siembras en un lapso de tres años, lo que implica mayor laboreo del suelo (Figura 4). En este tipo de suelos el mayor laboreo conduce a la disminución de la estabilidad estructural del suelo que aumenta los niveles de erosión (Volverás et al., 2009).

Las menores pérdidas de suelo en floración en agosto y en madurez en septiembre $\left(0,83\right.$ y $\left.0,26 \mathrm{t} \mathrm{ha}^{-1}\right)$ se explican en gran parte en el hecho que, durante esos periodos de cultivo, no se realizaron labores drásticas de remoción de suelo. En efecto, si hubo que controlar malezas que aparecieron principalmente en los bordes del entresurco, se realizó con herbicida o con machete, labores que igual generaron desprendimiento de suelo hacia el canal del entresurco pero que fue menor; en cosecha la pérdida de suelo volvió a aumentar $\left(15,82 \mathrm{t} \mathrm{ha} \mathrm{a}^{-1}\right)$, debido a que se utilizó de nuevo la herramienta manual con remoción total del surco y a que la precipitación también aumentó (Figura 6).

La disminución de la erosión obtenida también al reducir el grado de inclinación del surcado de 20,45 t ha ${ }^{-1}$ en labranza comercial a $90^{\circ}$ a $10,60, \mathrm{t} \mathrm{ha}^{-1}(48 \%)$ en wachado con surcos a $60^{\circ}$ en cosecha, estuvo relacionada con el efecto combinado de la pendiente y el laboreo del suelo; al inclinarse el surco, la escorrentía pierde energía y velocidad que disminuye el arrastre de suelo, resultados similares a los reportados por Ordoñez (2007) en un estudio sobre surcado en wachado en Nariño, quien encontró una disminución del 59 \% en la erosión del suelo al pasar de surcado en el sentido de la pendiente a surcado a $45^{\circ}$.

La disminución obtenida al reducir el grado de inclinación del surcado (hasta $60 \%$ en cosecha) al pasar de surcado en el sentido de la pendiente a surcado a $60^{\circ}$ y $70^{\circ}$, estuvo relacionada con la pérdida de energía y velocidad de la escorrentía, tal como lo reportaron Shanshan et al. (2018), quienes en una compilación de información sobre escorrentía y erosión en laderas, corroboraron el efecto de la intensidad de la lluvia y la gradiente de la pendiente que determinaron el nivel de energía erosiva que causaba la erosión del suelo.

El efecto de la localidad por época de evaluación sobre la pérdida de suelo estuvo ligado a aspectos como condiciones de suelo, condiciones topográficas, condiciones ambientales y climáticas; los tres eran suelos Andisoles con régimen de humedad údico y se diferenciaban un poco a nivel de gran grupo; en general, eran suelos con baja densidad aparente, típica de estos sin o con baja mecanización intensiva, con dominio de agregados menores a 1,0 $\mathrm{mm}$ (Campo et al., 2014).

La alta pérdida total de suelo durante todo el ciclo de cinco meses como sumatoria de la pérdida en cada una de las épocas, es de particular interés en cada nivel de inclinación de surcado como alternativa técnica para disminuir el proceso erosivo. La pérdida acumulada en parte se puede atribuir a diferencias en volumen de lluvias durante los ciclos de cultivo por localidad, la época de siembra, el grado de inclinación, el tipo de pendiente y la labranza que, en ladera, puede contribuir más a la erosión que la lluvia (Rymshaw et al., 1997). Lo anterior concuerda con las consideraciones de Mehuys et al. (2009), quienes determinaron la relación entre escorrentía, labranza y pendiente, de tal manera que la escorrentía generó pérdida de suelo en las partes media y baja de la pendiente y la labranza 
causó pérdida de suelo desde la parte superior de la pendiente; de la misma forma Ruiz et al. (2002), reportaron una pérdida de suelo $63 \%$ mayor en wachado con surcado en el sentido de la pendiente frente a sistemas de labranza mínimas o reducidas.

Las pérdidas totales entre 45,62 y 75,83 $\mathrm{t} \mathrm{ha}^{-1}$ en wachado con surcados a favor de la pendiente y entre 32,53 y $65,23 \mathrm{t} \mathrm{ha}^{-1}$ en los surcados a $60^{\circ}$ y $70^{\circ}$ fueron consideradas altas para un sistema donde por años no se usa maquinaria pesada, esto puede estar asociados a la práctica de aflojamiento del suelo al inicio de las temporadas de lluvia (abril, mayo). Según la Organización para la Alimentación y la Agricultura (Food and Agriculture Organization (2015) y el Instituto de Hidrología, Meteorología y Estudios Ambientales (2014) en estudios sobre la situación actual de la degradación del suelo en el mundo y en Colombia, una pérdida de suelo mayor a 30 t ha $^{-1}$ año ${ }^{-1}$ genera una erodabilidad de severa a muy severa. Los resultados de este estudio concuerdan con los reportes de AlTaai et al. (2016), quienes determinaron una fuerte relación positiva entre la lluvia y la erosión con mayor impacto en terrenos inclinados, asimismo, se reportó que la sola operación de labranza puede trasladar instantáneamente el suelo de 15 a $30 \mathrm{~cm}$ y una estrecha relación entre la erosión por labranza y la erosión hídrica que pueden actuar conjuntamente (Mehuys et al., 2009).

Los resultados obtenidos en el estudio estuvieron relacionados con algunas condiciones pedológicas y agronómicas. Características del suelo como bajo volumen $\left(700 \mathrm{~kg} \mathrm{~m}^{-3}\right)$, buen contenido de materia orgánica $(\sim 14$ $\%$ ), buena profundidad, hacen que el suelo sea muy susceptible a efectos de la labranza y la lluvia, lo cual explica en gran medida las mayores pérdidas de suelo en las épocas de mayor laboreo. Un aspecto agronómico que favorece la pérdida de suelo bajo las condiciones particulares de cada localidad, es el repique del suelo, la formación del canal y la ubicación del suelo suelto sobre el lomo del surco.

El rendimiento total de tubérculo, que fue un $40 \%$ superior al rendimiento en labranza mecanizada intensiva en todos los tratamientos en las tres localidades, puede estar asociado a los altos contenidos de materia orgánica, fósforo, potasio y contenidos medios de azufre, molibdeno y zinc. Otro aspecto que influyó en los rendimientos está asociado a buenas características físicas del suelo como baja densidad aparente, mejor capacidad de regulación de humedad y mayor abundancia y riqueza de macroorganismos, sostenidas por el manejo en wachado por veinticinco años o más, donde no se invierte el suelo y no se expone al efecto de implementos y máquinas pesadas; en efecto, según Campo et al. (2014), los suelos bajo wachado presentan densidad aparente menor a $0,7 \mathrm{~g} \mathrm{cc}^{-1}$, una capacidad de retención de humedad entre $73 \%$ y $45 \%$ para baja y alta succión, una porosidad total mayor al $74 \%$ y profundidad entre 1,0 y $1,5 \mathrm{~m}$.

\section{Conclusiones}

En el sistema de siembra en wachado no se utiliza maquinaria ni implementos pesados, pero se presentó alta pérdida de suelo, por lo cual es necesario realizar ajustes en las labores agronómicas donde se presentó la mayor pérdida.

La disminución en la inclinación del surcado tuvo efecto significativo en la disminución de la pérdida de suelo, aspecto que debe ser objeto de más evaluaciones para una inclinación que disminuya aún más la pérdida de suelo sin afectar la operabilidad de la pala de cute y la eficiencia del operario al realizar el surcado en wachado en pendientes pronunciadas.

En el sistema de siembra en wachado se perdió menos suelo que en los sistemas con mecanización intensiva y se mantuvo mejores indicadores de fertilidad, por lo cual debe ser objeto de estudios en el componente agrícola y pecuario que garanticen su permanencia en el tiempo en laderas escarpadas donde se practica. 


\section{Agradecimientos}

Los autores expresan sus agradecimientos a la Corporación Colombiana de Investigación Agropecuaria (AGROSAVIA) y al Ministerio de Agricultura y Desarrollo Rural (MADR) de Colombia quien financió con recursos públicos el macroproyecto en el que se realizó esta investigación.

\section{Referencias}

Al-Taai, O. T., Al-Hassani, D. A., \& Mehdi, A. M. (2016). Estimating the soil erosion by using rainfall data for selected stations in Iraq. Open Access Library Journal, 3, e2494. http://dx.doi.org/10.4236/oalib.1102494

Amézquita, E. (1999). Propiedades físicas de los suelos de los Llanos Orientales y sus requerimientos de labranza. Revista Palmas, 20(1), 73-86. https://publicaciones.fedepalma.org/index.php/palmas/article/view/692

Bastidas, S. (2003). Evaluación de alternativas metodológicas para el Manejo Integrado de Suelo en el cultivo de la papa en la provincia del Carchi [Tesis de pregrado, Pontifica Universidad Católica del Ecuador]. Repositorio de la Universidad Politécnica Estatal del Carchi. http://repositorio.upec.edu.ec/bitstream/123456789/764/1/348\%20Alternativas\%20 de\%20fertilizaci\%C3\%B3n\%20para\%20el\%20cultivo\%20de\%20papa\%20-\%20Mont\%C3\%BAfar.pdf

Campo, J.M., Volverás, B., Pérez, O., \& Sánchez, G. (2014, 28 de septiembre - 2 de octubre). Condición física del suelo andino bajo el sistema de siembra de papa en guachado (wachado) (p. 81). [Memorias XXVI Congreso de la Asociación Latinoamericana de la Papa (ALAP)]. Asociación Latinoamericana de la Papa, Bogotá, Colombia. http://www. papaslatinas.org/alap2014/

Colotti, E. (1999). La erosividad: cualidad de la lluvia poco conocida. Terra Nueva Etapa, 15(24), 99-116. https://www.redalyc. org/pdf/721/72102406.pdf

Fondo Nacional de Fomento de la Papa. (2016). Informe de gestión 2016. Fondo Nacional de Fomento de la Papa. https:// fedepapa.com/wp-content/uploads/2017/01/INFORME-DE-GESTIO\%CC\%81N-FNFP-ANUAL-2016.pdf

Food and Agriculture Organization. (2000). Sistemas Importantes del Patrimonio Agrícola Mundial (SIPAM). http://www.fao. org/3/19187es/I9187ES.pdf

Food and Agriculture Organization. (2010). Sistematización de buenas prácticas en el marco de la prevención y mitigación de siniestros climáticos en el sector agropecuario. Caso territorio indígena Jach'a Suyu Pakajaqi en el altiplano central y Yapuchiris en Omasuyos, altiplano norte, Bolivia. http://www.fao.org/3/al930s/a1930s.pdf

Food and Agriculture Organization. (2015). Suelos y biodiversidad. Los suelos albergan una cuarta parte de la biodiversidad de nuestro planeta. 2015, Año Internacional de los Suelos. http://www.fao.org/3/a-i4551s.pdf

Graves, C. (Ed.). (2006). La papa tesoro de los andes (2da ed.). Centro Internacional de la Papa. http://cipotato.org/wp-content/ uploads/2014/05/la_papa_tesoro_de_los_andess.pdf

Holdridge, L. R. (1987). Ecología basada en zonas de vida. Instituto Interamericano de Ciencias Agrícolas. https://repositorio. iica.int/bitstream/handle/11324/7936/BVE19040225e.pdf?sequence=1\&isAllowed=y

Instituto Colombiano de Normas Técnicas y Certificación (1996). Norma técnica colombiana NTC 341: papa consumo, clasificación (pp. 49-54). Federación Colombiana de Productores de Papa. https://fedepapa.com/wp-content/ uploads/2017/01/Normatividad-del-Sector-de-la-Papa.pdf 
Instituto de Hidrología, Meteorología y Estudios Ambientales (IDEAM). (2014, 10 de diciembre). Solicitud de información meteorológica. http://www.ideam.gov.co/solicitud-de-informacion/

Instituto Geográfico Agustín Codazzi. (2004). Descripción de los suelos. En: L. Burgos (Ed.), Estudio general de suelos y zonificación de tierras: Departamento de Nariño (p. 61-168). Instituto Geográfico Agustín Codazzi.

Lal, R. (1993). Tillage effects on soil degradation, soil resilience, soil quality, and sustainability. Soil and Tillage Research, 27, 1-8. https://doi.org/10.1016/0167-1987(93)90059-X

Mehuys, G.,Tiessen, K.,Villatoro, M.,Sancho, F., y Lobb, D. (2009). Erosión por labranza con arado de disco en suelos volcánicos de ladera en Costa Rica. Agronomía Costarricense, 33(2), 205-215. https://www.mag.go.cr/rev_agr/ v33n02_205.pdf

Moore, T. R. (1979). Rainfall Erosivity in East Africa. Geografiska Annaler: Series A, Physical Geography, 61(3-4), 147-156. https://doi.org/10.1080/04353676.1979.11879987

Ordoñez, E. (2007). Efecto del sistema guachado (wachay) y uso del suelo sobre algunas propiedades físicas en la microcuenca del río Bobo, departamento de Nariño [Tesis de maestría, Universidad Nacional de Colombia en Convenio con la Universidad de Nariño]. Repositorio de la Universidad Nacional de Colombia. https://repositorio.unal.edu.co/ bitstream/handle/unal/2460/eduardoordonezcastillo.2007.pdf?sequence=1\&isAllowed=y

Pumisacho, M., \& Sherwood, S. (Ed.) (2002). El cultivo de papa en Ecuador. Centro Internacional de la papa. https://cipotato. org/wp-content/uploads/Documentacion\%20PDF/Pumisacho\%20y\%20Sherwood\%20Cultivo\%20de\%20Papa\%20 en\%20Ecuador.pdf

Ramos, M., Córdova, J., Valverde, F., Reinoso, L., \& Oyarzun, P. (2002, 26 - 27 de septiembre). Evaluación de tres sistemas de labranza del suelo en el cultivo de papa, con fines de producción de tubérculo-semilla [Memorias conferencia VIII Congreso Ecuatoriano de la Ciencia del suelo] Instituto Nacional Autónomo de Investigaciones Agropecuarias. http:// www.secsuelo.org/wp-content/uploads/2015/06/4-Evaluacion-de-tres-sistemas-de-labranza-de-papa-Ramos-M.pdf

Red de Información y Comunicación del Sector Agropecuario de Colombia. (2019, 29 de noviembre). Reporte: área, producción y rendimiento nacional por cultivo (papa). Informe estadísticas. https://www.agronet.gov.co/estadistica/ Paginas/home.aspx ?cod=1

Rozas, J. (2007). El modo de pensar andino: Una interpretación de los rituales de Calca [Tesis de maestría, Pontificia Universidad Católica del Perú]. Repositorio de la Pontificia Universidad Católica del Perú. http://tesis.pucp.edu.pe/ repositorio/handle/20.500.12404/1409

Ruiz, H., Legarda, L., Amézquita, E., Arteaga, G., Ceballos, D., \& Hernández, O. (2002). Dinámica de la erosión del suelo bajo cuatro sistemas de labranza, cuantificada a través del microrelievímetro en suelos paperos de Nariño, Colombia. Revista de Ciencias Agricolas, 19(1), 23-36. https://revistas.udenar.edu.co/index.php/rfacia/article/view/928

Rymshaw, E., Walter, M. F., \& Van-Wambeke, A. (1997). Processes of soil movement on steep cultivated hill slopes in the Venezuelan Andes. Soil and Tillage. Research, 44(3), 265-272.https://doi.org/10.1016/S0167-1987(97)00055-X

Sánchez, F. (2009). Características de los sistemas agroecológicos que incluyen estrategias de agricultura de coberturas, en las localidades que constituyen la zona de acción de la red Macrena, aplicando la metodología para la evaluación de sistemas de manejo incorporando indicadores de sustentabilidad [Tesis no publicada de pregrado]. Pontificia Universidad Católica del Ecuador. 
Shanshan, W., Baoyang, S., Chaodong, L., Zhanbin, L., \& Bo, M. (2018). Runoff and soil erosion on slope Cropland: A Review. Journal of Resources and Ecology, 9(5), 461-470. https://doi.org/10.5814/j.issn.1674-764x.2018.05.002

Stadel, C. (2005). Cultivated landscapes of native Amazonia and the Andes. Mountain Research and Development, 25(4), 388-389. https://10.2307/3674449

Thompson, L. M., \& Troeh, F. R. (2009). Los suelos y su fertilidad (4ta ed.). Editorial Reverté.

Toapanta, G. (2004). Evaluación socioeconómica del sistema de labranza reducido wachu rozado frente a dos sistemas de labranza en el cultivo de papa, en la provincia del Carchi [Tesis no publicada de pregrado]. Universidad Central del Ecuador.

Valdez, F. (1987). Proyecto arqueológico La Tolita. Fondo arqueológico del Museo del Banco Central Guillermo Pérez Chiriboga.

Volverás, B., \& Amézquita, E. (2009). Estabilidad estructural del suelo bajo diferentes sistemas y tiempos de uso en laderas andinas de Nariño, Colombia. Acta Agronómica, 58(1), 35-40. https://revistas.unal.edu.co/index.php/acta_ agronomica/article/view/9741

Volverás, B., Amézquita, E., \& Campo J. M. (2016). Indicadores de calidad física del suelo de la zona cerealera andina del departamento de Nariño, Colombia. Ciencia y Tecnología Agropecuaria, 17(3), 361-377. https://doi.org/10.21930/ rcta.vol17_num3_art:513

Volverás, B., Amézquita, E., \& Tafurt, H. (2007). Efecto del tiempo de uso en las condiciones físicas del suelo Andino en el departamento de Nariño, Colombia. Acta Agronómica, 56(1), 29-36. https://revistas.unal.edu.co/index.php/acta_ agronomica/article/view/566

Volverás, B., Campo, J. M., Pérez, O., \& Sanchez, G. (2014, 28 de septiembre - 2 de octubre). El sistema de siembra de papa en guachado (wachado) en la zona altoandina de Nariño (p. 82). [Memorias XXVI Congreso de la Asociación Latinoamericana de la Papa (ALAP)]. Asociación Latinoamericana de la Papa. http://www.papaslatinas.org/alap2014/

Wischmeier, W. H., \& Smith, D. (1978). Predicting rainfall erosion losses. A guide to conservation planning. United States Departmen of Agriculture.

Yapa, K. (2013). Prácticas ancestrales de crianza de agua, una guía de campo. Estrategias para adaptarnos a la escasez de agua. Reimpresión Edipcentro Cía. Ltda. 